\title{
Global NLO Analysis of Nuclear Parton Distribution Functions
}

\author{
M. Hirai*, S. Kumano ${ }^{\dagger, * *}$ and T.-H. Nagai** \\ ${ }^{*}$ Department of Physics, Juntendo University, Inba, Chiba, 270-1695, Japan \\ ${ }^{\dagger}$ Institute of Particle and Nuclear Studies, High Energy Accelerator Research Organization (KEK) \\ 1-1, Ooho, Tsukuba, Ibaraki, 305-0801, Japan \\ ${ }^{* *}$ Department of Particle and Nuclear Studies, Graduate University for Advanced Studies \\ 1-1, Ooho, Tsukuba, Ibaraki, 305-0801, Japan
}

\begin{abstract}
Nuclear parton distribution functions (NPDFs) are determined by a global analysis of experimental measurements on structure-function ratios $F_{2}^{A} / F_{2}^{A^{\prime}}$ and Drell-Yan cross section ratios $\sigma_{D Y}^{A} / \sigma_{D Y}^{A^{\prime}}$, and their uncertainties are estimated by the Hessian method. The NPDFs are obtained in both leading order (LO) and next-to-leading order (NLO) of $\alpha_{s}$. As a result, valence-quark distributions are relatively well determined, whereas antiquark distributions at $x>0.2$ and gluon distributions in the whole $x$ region have large uncertainties. The NLO uncertainties are slightly smaller than the LO ones; however, such a NLO improvement is not as significant as the nucleonic case.
\end{abstract}

Keywords: Parton, distribution, quark, gluon, nucleus

PACS: $13.60 . \mathrm{Hb}, 12.38 .-\mathrm{t}, 24.85 .+\mathrm{p}, 25.30 .-\mathrm{c}$

\section{INTRODUCTION}

From measurements on structure-function ratios $F_{2}^{A} / F_{2}^{D}$, we found that nuclear parton distribution functions (NPDFs) are not equal to corresponding nucleonic PDFs. Although there are many analyses on the PDFs in the nucleon, the NPDFs have not been investigated extensively. However, such studies are becoming more and more important in recent years in order to precisely understand measurements of heavy-ion reactions at RHIC and LHC. It should lead to a better understanding on properties of quark-hadron matters.

Such investigations are also valuable for applications to neutrino reactions. For an accurate determination of neutrino oscillation, nuclear corrections in the oxygen nucleus are important. Although the oscillation experiments are done in a relatively low-energy region, gross properties of cross sections could be described by using quark-hadron duality. In future, nuclear structure functions will be investigated by the MINER $v$ A project and at neutrino factories.

The unpolarized PDFs in the nucleon have been investigated for a long time, and they are relatively well determined. Uncertainties of the determined PDFs were also estimated recently, and such studies were extended to the polarized PDFs [1] and fragmentation functions [2]. On the other hand, we have been investigating parametrization of the NPDFs and their uncertainties in a similar technique $[3,4]$ by analyzing experimental data on nuclear structure-function ratios $F_{2}^{A} / F_{2}^{A^{\prime}}$ and Drell-Yan cross section rations $\sigma_{D Y}^{A} / \sigma_{D Y}^{A^{\prime}}$. However, the uncertainty estimation was limited to the leading order (LO) in the previous analysis [4]. It is the purpose of this work to show the NPDFs and their uncertainties in both LO and next-to-leading order (NLO) and to discuss NLO improvements [5].

\section{ANALYSIS METHOD}

First, the functional form of our NPDFs is introduced. Since nuclear modifications are generally within the $10 \%-30 \%$ range for medium and large size nuclei, it is easier to investigate the modifications than the absolute NPDFs. We define the NPDFs at the initial $Q^{2}$ scale $\left(\equiv Q_{0}^{2}\right)$ as

$$
f_{i}^{A}\left(x, Q_{0}^{2}\right)=w_{i}(x, A, Z) f_{i}\left(x, Q_{0}^{2}\right),
$$

where $f_{i}^{A}\left(x, Q_{0}^{2}\right)$ is the parton distribution with type $i$ $\left(=u_{v}, d_{v}, \bar{u}, \bar{d}, s, g\right)$ in a nucleus, $f_{i}\left(x, Q_{0}^{2}\right)$ is the corresponding parton distribution in the nucleon, $A$ is mass number, and $Z$ is the atomic number. The variable $Q^{2}$ is given $Q^{2}=-q^{2}$ with the virtual photon momentum $q$ in lepton scattering, and the Bjorken variable $x$ is defined by $x=Q^{2} /(2 M v)$ with the energy transfer $v$ and the nucleon mass $M$. We call $w_{i}(x, A, Z)$ a weight function, which indicates a nuclear modification for the type- $i$ distribution. The weight functions are expressed by

$$
w_{i}(x, A, Z)=1+\left(1-\frac{1}{A^{\alpha}}\right) \frac{a_{i}+b_{i} x+c_{i} x^{2}+d_{i} x^{3}}{(1-x)^{\beta_{i}}},
$$


where $\alpha, a_{i}, b_{i}, c_{i}, d_{i}$, and $\beta_{i}$ are parameters to be determined by a $\chi^{2}$ analysis. Here, the valence up- and downquark parameters are the same except for $a_{u_{v}}$ and $a_{d_{v}}$. Since there is no data to find flavor dependence in antiquark modifications, the weight functions of $\bar{u}, \bar{d}$, and $\bar{s}$ are assumed to be the same at $Q_{0}^{2}$. We impose three conditions, baryon-number, charge, and momentum conservations, so that three parameters are fixed.

The initial scale of the NPDFs is chosen $Q_{0}^{2}=1 \mathrm{GeV}^{2}$. They are evolved to experimental $Q^{2}$ points. From the threshold at $Q^{2}=m_{c}^{2}$, charm-quark distributions appear due to the $Q^{2}$ evolution. Using these NPDFs, we calculate $F_{2}^{A} / F_{2}^{A^{\prime}}$ and Drell-Yan ratios $\sigma_{D Y}^{A} / \sigma_{D Y}^{A^{\prime}}$. The parameters are determined so as to minimize the total $\chi^{2}$

$$
\chi^{2}=\sum_{j} \frac{\left(R_{j}^{\text {data }}-R_{j}^{\text {theo }}\right)^{2}}{\left(\sigma_{j}^{\text {data }}\right)^{2}},
$$

where $R_{j}$ indicates $F_{2}^{A} / F_{2}^{A^{\prime}}$ and $\sigma_{D Y}^{A} / \sigma_{D Y}^{A^{\prime}}$. The uncertainties of the determined NPDF are estimated by the Hessian method:

$$
\left[\delta f^{A}(x)\right]^{2}=\Delta \chi^{2} \sum_{i, j} \frac{\partial f^{A}(x, \hat{\xi})}{\partial \xi_{i}} H_{i j}^{-1} \frac{\partial f^{A}(x, \hat{\xi})}{\partial \xi_{j}}
$$

where $H_{i j}$ is the Hessian matrix, $\xi_{i}$ is a parameter, and $\hat{\xi}$ indicates the optimum parameter set. The $\Delta \chi^{2}$ value is taken as 13.7 so that the confidence level $P$ becomes the one- $\sigma$-error range $(P=0.6826)$ for thirteen parameters by assuming the normal distribution in the multiparameter space.

\section{RESULTS}

We used data with $Q^{2} \geq 1 \mathrm{GeV}^{2}$. They consist of 290 , 606, 293, and 52 data points for $F_{2}^{D} / F_{2}^{p}, F_{2}^{A} / F_{2}^{D}$, $F_{2}^{A} / F_{2}^{A^{\prime}}\left(A^{\prime} \neq D\right)$, and $\sigma_{D Y}^{A} / \sigma_{D Y}^{A^{\prime}}$, respectively. For the total 1241 data, we obtained the minimum $\chi^{2}$ values, $\chi_{\min }^{2} /$ d.o.f. $=1.35$ and 1.21 for the LO and NLO.

As examples, we show actual data with the theoretical LO and NLO ratios for the calcium nucleus together with their uncertainties in Figs. 1 and 2. The theoretical curves and their uncertainties are calculated at $Q^{2}=$ $10 \mathrm{GeV}^{2}$ and $Q^{2}=50 \mathrm{GeV}^{2}$ for the $F_{2}$ and the Drell-Yan, respectively; however, the experimental data were taken at various $Q^{2}$ values. There are discrepancies from the experimental data at $x<0.01$ in Fig. 1, but they should be attributed to the $Q^{2}$ difference [5]. The figures indicate good agreement between the data and the theoretical curves, and most data are within the uncertainties. As expected, the NLO uncertainties are smaller than the LO ones, especially at small $x$, but they are similar in the region, $x>0.02$. As for Drell-Yan ratios in the region

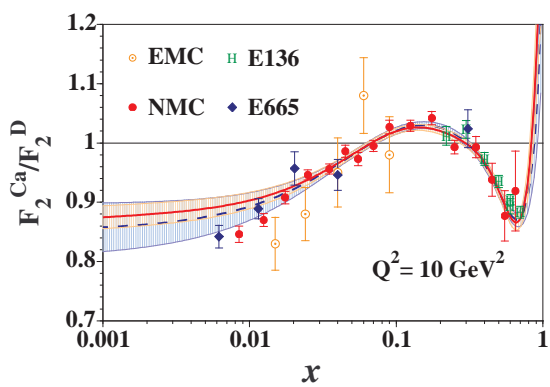

FIGURE 1. Experimental data of $F_{2}^{C a} / F_{2}^{D}$ are compared with theoretical ratios, which are calculated at $Q^{2}=10 \mathrm{GeV}^{2}$. The dashed and solid curves indicate LO and NLO results, and the uncertainties are shown by the shaded bands.

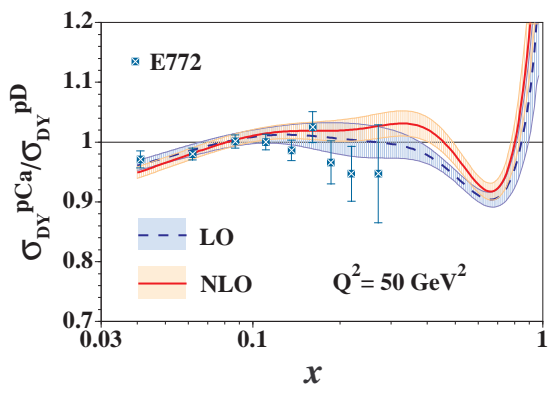

FIGURE 2. Experimental data of $\sigma_{D Y}^{p C a} / \sigma_{D Y}^{p D}$ are compared with the theoretical ratios calculated at $Q^{2}=50 \mathrm{GeV}^{2}$. The dashed and solid curves indicate LO and NLO results, and the uncertainties are shown by the shaded bands.

$x>0.04$, the NLO effects on the uncertainties are not obvious.

In order to illustrate the nuclear dependence, we show the weight functions for all the analyzed nuclei and ${ }^{16} \mathrm{O}$ at $Q^{2}=1 \mathrm{GeV}^{2}$ in Fig. 3. The NPDFs in the oxygen nucleus are shown because they are important for neutrinooscillation studies. As the mass number becomes larger in the order of $\mathrm{D},{ }^{4} \mathrm{He}, \mathrm{Li}, \cdots$, and $\mathrm{Pb}$, the curves deviate from the line of unity.

We provide a code for calculating the NPDFs and their uncertainties at our web site [6]. By supplying the kinematical conditions, $x$ and $Q^{2}$, and a nuclear species, one can obtain the NPDFs $\left(u^{A}, d^{A}, s^{A}, \bar{u}^{A}, \bar{d}^{A}, s^{A}, c^{A}\right.$, and $g^{A}$ ) numerically. The technical details on its usage are explained in Refs. $[4,5]$ and within the subroutine.

Nuclear modifications and their uncertainties are shown for the calcium nucleus in Fig. 4. The valencequark distributions are well determined in the medium and large $x$ regions because the $F_{2}$ structure functions are dominated by the valence-quark distributions and because the $F_{2}$ ratios are accurately measured. At small $x$, the valence-quark modifications have also small uncertainties because of the baryon-number and charge 

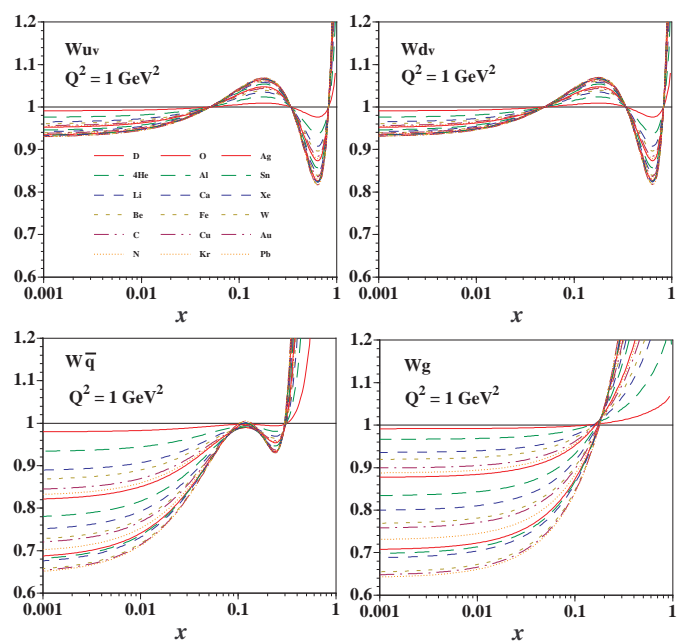

FIGURE 3. Nuclear modifications $w_{i}\left(i=u_{v}, d_{v}, \bar{q}\right.$, and $g$ ) are shown in the NLO for all the analyzed nuclei and ${ }^{16} \mathrm{O}$ at $Q^{2}=1 \mathrm{GeV}^{2}$.

conservations.

The antiquark distributions are well determined at $x<$ 0.2 due to the $F_{2}$ and Drell-Yan data. However, they have large uncertainties at $x>0.2$ because there is no DrellYan data which constrains the antiquark modifications. Future Drell-Yan measurements at large $x$ should improve the situation of the antiquark distributions. There are J-PARC, Fermilab-E906, and GSI-FAIR projects, in which Drell-Yan processes will be investigated.

We found that the uncertainty bands for the gluon are very large, which indicates that the gluon modifications
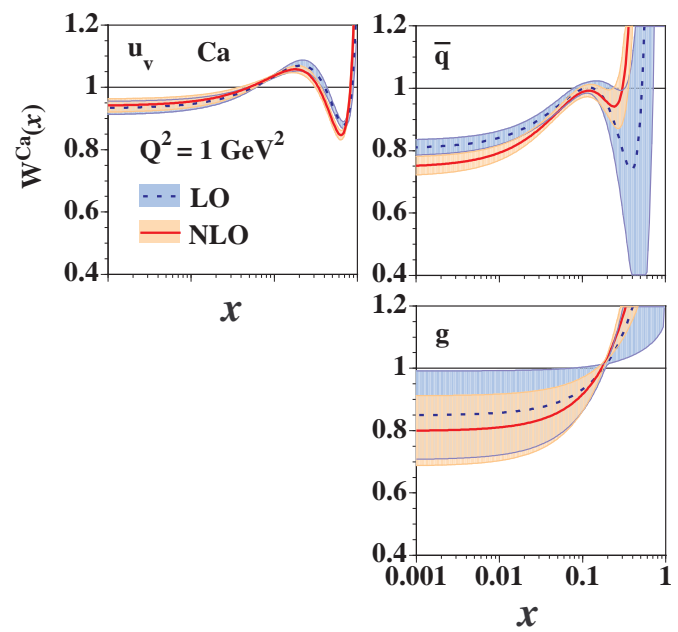

FIGURE 4. Nuclear modifications of the PDFs and their uncertainties are shown for the calcium nucleus at $Q^{2}=1 \mathrm{GeV}^{2}$. The dashed and solid curves indicate LO and NLO results, and their uncertainties are shown by the shaded bands. cannot be well determined in the whole $x$ region. The gluon distributions contribute to the $F_{2}$ and Drell-Yan ratios as higher-order effects. Therefore, the gluon distributions cannot be accurately determined especially in the LO analysis. Some improvements are expected in the NLO analysis. In fact, Fig. 4 indicates that the NLO uncertainty band for the gluon becomes smaller than the LO one. However, it is not as clear as the improvements in the polarized PDFs [1] and the fragmentation functions [2]. The gluon distribution in the nucleon has been determined mainly by using $Q^{2}$ dependence of the structure function $F_{2}$. In the nuclear case, the $Q^{2}$ dependencies of the ratios $F_{2}^{A} / F_{2}^{A^{\prime}}$ are not accurately measured. It leads to the large uncertainty bands in the gluon modifications even in the NLO analysis. We hope that accurate data will be provided at future electron facilities such as eRHIC and eLIC.

Nuclear modifications in the deuteron are also investigated in our recent analysis [5]. Since the deuteron data are used for determining the "nucleonic" PDFs after some nuclear corrections, current PDFs in the nucleon could partially contain nuclear effects. Proper nuclear corrections should be taken into account in the PDF analysis of the nucleon to exclude such effects.

\section{SUMMARY}

By the global analyses of the data on the nuclear $F_{2}$ and Drell-Yan ratios, the NPDFs have been determined in both LO and NLO. Although the valence-quark distributions and antiquark ones at $x<0.2$ are well determined, the uncertainty bands are very large in the antiquark modifications at $x>0.2$ and the gluon ones at whole $x$ even in the NLO analysis. We need future experimental efforts for determining all the nuclear modifications. Our NPDFs and their uncertainties can be calculated by the code supplied in Ref. [6].

\section{REFERENCES}

1. M. Hirai, S. Kumano, and N. Saito, Phys. Rev. D 69, 054021 (2004); 74, 014015 (2006).

2. M. Hirai, S. Kumano, T.-H. Nagai, and K. Sudoh, Phys. Rev. D 75, 094009 (2007); M. Hirai, S. Kumano, M. Oka, and K. Sudoh, arXiv:0708.1816 [hep-ph].

3. M. Hirai, S. Kumano, and M. Miyama, Phys. Rev. D 64, 034003 (2001).

4. M. Hirai, S. Kumano, and T.-H. Nagai, Phys. Rev. C 70, 044905 (2004).

5. M. Hirai, S. Kumano, and T.-H. Nagai, arXiv:0709.3038 [hep-ph].

6. Our code for the NPDFs is available at http://research.kek.jp/people/kumanos/nuclp.html. 